\title{
Enfermedad celíaca y el nuevo Coronavirus SARS-CoV-2
}

\author{
Alberto Espino E. ${ }^{1}$, Alejandra Parada D. ${ }^{2}$ y Helga Santibáñez $W^{3}$
}

\section{Celiac disease and the novel Coronavirus SARS-CoV-2}

To date, it has not been described that celiac disease (CD) increases the risk of contagion by the new coronavirus SARS-CoV-2 nor the severity of the disease. The main risk factors for severe disease for this coronavirus are old male patients ( $>65$ years) with hypertension, diabetes, obesity, smoking, lung, cardiovascular and kidney diseases. The only treatment for $\mathrm{CD}$ is a gluten-free diet that improves the intestinal immune response. Adherence to treatment and follow-up of recommendations by experts during the $\mathrm{CO}$ VID-19 period, would ensure celiac patients are better prepared for this pandemic.

Key words: SARS-CoV-2, coronavirus COVID-19, celiac disease.

\section{Resumen}

Hasta la fecha no se ha descrito que la enfermedad celíaca (EC) incremente el riesgo de contagio por el nuevo coronavirus SARS-CoV-2 ni la gravedad de la enfermedad. Los principales factores de riego de enfermedad grave para este coronavirus son pacientes mayores de 65 años de sexo masculino con hipertensión, diabetes, obesidad, tabaquismo, y enfermedades pulmonar, cardiovascular y renal. El único tratamiento de la EC es la dieta libre de gluten que mejora la respuesta inmune intestinal. La adherencia al tratamiento y el seguimiento de recomendaciones dadas por expertos durante el período COVID-19, aseguraría a los enfermos celíacos, estar mejor preparados para esta pandemia.

Palabras clave: SARS-CoV-2, coronavirus COVID-19, enfermedad celíaca.

\section{Introducción}

La enfermedad celíaca (EC) es una enfermedad sistémica autoinmune caracterizada por inflamación crónica y atrofia de la mucosa del intestino delgado por ingesta de gluten en personas genéticamente susceptibles ${ }^{1}$. Se estima que afecta entre el $0,3 \%$ y el $0,6 \%$ de la población chilena, con un predominio en el sexo femenino $(3: 1)^{2,3}$. Los factores ambientales pueden contribuir a la pérdida de tolerancia al gluten. Las infecciones podrían predisponer a la EC, induciendo la producción y liberación de la enzima transglutaminasa tisular ${ }^{4}$, la cual es importante para aumentar la inmunogenicidad del gluten. Además, el estado inflamatorio durante una infección puede promover la activación de células $\mathrm{T}$ espectadoras autoreactivas $^{5}$, que es un paso crítico en la patogénesis de EC. Un estudio de cohorte noruego basado en registros a nivel nacional encontró una asociación positiva modesta de influenza estacional e influenza pandémica con EC posterior, consistente con la hi- pótesis de que las alteraciones inmunes infecciosas juegan un papel en el desarrollo de EC en la infancia hasta la mediana edad ${ }^{6}$. Se ha descrito también que pacientes con EC pueden presentar un mayor riesgo de influenza grave y hospitalización ${ }^{7}$. El objetivo de este artículo es revisar los riesgos y posibles implicancias de la infección por el nuevo coronavirus SARS-CoV-2 (por sus siglas en inglés Severe Acute Respiratory Syndrome-CoV-2 $)^{8}$ causante de la pandemia COVID-19 (por sus siglas en inglés Coronavirus Disease 2019) en pacientes con EC y entregar recomendaciones para los pacientes.

\section{Enfermedad celíaca y riesgo de infección por coronavirus SARS-CoV-2}

La EC no diagnosticada se ha asociado a morbilidad y mortalidad. La normalización clínica, serológica e histológica ocurre típicamente después de una estricta dieta libre de gluten (DLG). Sin embargo, la

\author{
'Departamento de \\ Gastroenterología \\ Escuela de Medicina, \\ Pontificia Universidad \\ Católica de Chile. \\ Santiago, Chile. \\ 2Departamento \\ Nutrición, Diabetes \\ y Metabolismo, \\ Escuela de Medicina, \\ Pontificia Universidad \\ Católica de Chile. \\ Santiago, Chile. \\ 3Fundación de \\ Intolerancia al Gluten- \\ CONVIVIR.
}

Recibido:

Aceptado:

Correspondencia a: Dr. Alberto Espino E. Profesor Asistente Departamento de Gastroenterología Pontificia Universidad Católica de Chile Jefe Unidad Procedimientos Digestivos. Red Salud UC-Christus Diagonal Paraguay 362. 4to piso. Santiago, Chile. aoespino@uc.cl 
mayoría de los estudios han demostrado que incluso después del diagnóstico, la EC se asocia con un aumento de mortalidad. Este aumento del riesgo se ha atribuido a las múltiples morbilidades asociadas, incluida las neoplasias linfoproliferativas, fracturas osteoporóticas, diabetes mellitus tipo $1 \mathrm{y}$ otras condiciones ${ }^{9}$.

En relación con la pandemia COVID-19 causada por la infección del virus SARS-CoV-2, es importante conocer que su presentación clínica puede variar desde pacientes asintomáticos, con síntomas respiratorios leves inespecíficos, a una disfunción de órganos grave, como el síndrome de dificultad respiratoria aguda (SDRA) que puede conducir a la muerte ${ }^{10}$. Los síntomas en la mayoría de los casos $(+80 \%)$ son leves, siendo los más comunes fiebre (83-98\%), tos (46$82 \%)$, mialgia/fatiga (11-44\%) y disnea $(31 \%)^{10,11}$. También se han descrito síntomas gastrointestinales: anorexia $27 \%$, diarrea $12 \%$, náuseas/vómitos $10 \%$, y dolor abdominal $9 \%{ }^{12-14}$. En Chile, se ha descrito diarrea en un 7,3\%, y dolor abdominal 3,7\% de los $\operatorname{casos}^{15}$. El principal mecanismo fisiopatológico propuesto es por el ingreso del SARS-CoV-2 al enterocito, utilizando la Enzima Convertidora de Angiotensina 2 (ECA2) como receptor, el cual es ampliamente distribuido a lo largo del tracto gastrointestinal ${ }^{16}$. Es importante prestar atención a las manifestaciones gastrointestinales, ya que son comunes y más frecuentes en pacientes graves. Dentro de los factores de riesgo para desarrollar enfermedad grave se han descrito pacientes mayores de 65 años de sexo masculino con comorbilidades crónicas: hipertensión, diabetes, obesidad, tabaquismo, enfermedades pulmonar, cardiovascular y renal ${ }^{10,11,17,18}$. Hasta el momento, no se ha descrito que el padecer EC incremente el riesgo de contagio por SARS-CoV-2 ni la gravedad de la enfermedad. Actualmente, para responder mejor esta pregunta se están recopilando datos en un registro internacional de pacientes adultos y pediátricos celíacos diagnosticados con COVID-19 llamado Surveillance Epidemiology of Coronavirus Under Research Exclusion (SECURE-Celiac), el cual está disponible en el sitio https://www.covidceliac.com/home. Además, en el futuro será importante determinar si la incidencia de EC aumenta por efecto de la pandemia COVID-19, tal como se ha hipotetizado en otras infecciones ${ }^{19}$. Las principales organizaciones y fundaciones internacionales de EC junto con sus asesores de expertos afirman que los pacientes con EC en general no deben ser considerados inmunodeprimidos ${ }^{20,21}$. Sin embargo, una pequeña proporción de pacientes celíacos que presenten algunas de las siguientes condiciones como: desnutrición intensa, pérdida de peso, EC refractaria tipo 2, usuarios de medicamentos inmunosupresores o con otras enfermedades graves, podrían tener un mayor riesgo de enfermedad grave por COVID-1922, sin embargo, no se disponen datos que respalden esta condición ${ }^{17,18}$. En el contexto de pacientes con EC que estén recibiendo tratamiento inmunosupresor y/o corticoides es altamente recomendable seguir estrictamente todas las recomendaciones de cuarentena, distanciamiento social, lavado de manos, uso adecuado de mascarilla y cambios del comportamiento de la población, todas estas intervenciones se han asociado con una reducción en la transmisión de COVID-19 en Hong Kong, y también es probable que hayan reducido sustancialmente la transmisión de influenza ${ }^{23}$. Por otra parte, estos pacientes no deberían suspender ni modificar su tratamiento inmunosupresor, siempre supervisado por su médico tratante ${ }^{18}$.

Se sabe además que un subgrupo de celíacos que presenta riesgo levemente aumentado de contraer infecciones con otros virus, como el virus influenza y el Herpes zoster, o de desarrollar neumonía por neumococo. Esto asociado a factores como deficiencia de la función del bazo (hipoesplenismo), desnutrición o déficit de vitamina $\mathrm{D}$, alteraciones en la permeabilidad de la mucosa intestinal, y disbiosis (cambios en la composición, diversidad y/o función de la microbiota intestinal). Todos estos factores podrían agravarse cuando los pacientes no se adhieren de forma estricta a la dieta sin gluten y no han tenido un adecuado control médico y nutricional ${ }^{1,2,9,20-22}$.

\section{Recomendaciones prácticas para pacientes con EC en tiempos de COVID-19}

- El único tratamiento de la EC es la dieta libre de gluten que mejora la respuesta inmune intestinal. La adherencia rigurosa y estricta al tratamiento en tiempos de COVID-19 asegura a los enfermos celíacos estar mejor preparados para esta pandemia y no presentar mayores riesgos de contagio ni gravedad en comparación al resto de la población. Los principales factores de riego de enfermedad grave para este coronavirus son pacientes mayores de 65 años de sexo masculino con hipertensión, diabetes, obesidad, tabaquismo, enfermedades pulmonar, cardiovascular y renal.

- No es el momento de realizar transgresiones alimentarias, ya que su salud se verá perjudicada, y por la dificultad de asistir a consultas médicas o urgencias. En caso de necesitar evaluación médica o nutricional prefiera la alternativa de telemedicina.

- Aliméntese de forma adecuada para prevenir déficit o excesos nutricionales, en especial en este período de ansiedad y disminución de actividad física, que favorece la ganancia de peso.

- Adecúe una rutina de ejercicios consistente el tiempo dentro de su casa. 
- Siempre lave sus manos con agua y jabón (al menos 20 segundos) antes de: preparar y consumir alimentos, después de manipular o preparar alimentos crudos, residuos y haber realizado las tareas de limpieza, al sonarse la nariz, estornudar o toser, después de usar el baño, después de comer, beber y/o fumar y manipular dinero ${ }^{24}$.

- Limpie o desinfecte sus áreas de trabajo (mesones) y utensilios antes de utilizarlos y al terminar el trabajo.

- Cuando salga a comprar alimentos evite ir acompañado, use adecuadamente mascarilla, mantenga y respete la distancia con otros compradores y con el vendedor.

- Evite saludar de mano y el contacto físico, mantenga la distancia social hasta que las autoridades sanitarias lo recomienden.

- Evite compras innecesarias y en grandes cantidades, el sobreabastecimiento aumenta los desperdicios de alimentos y perjudica a otras personas.

- Al comprar alimentos y llegar a su casa, lave sus manos con agua y jabón y limpie los envases de los alimentos con desinfectantes (solución clorada o un paño con clorogel). Guarde y almacene los alimentos sin gluten de forma separada y bien identificada.

- Lave las frutas y verduras con solución de cloro (1 cucharada sopera de cloro líquido para un litro de agua fría).

- Lave y desinfecte las bolsas reutilizables o del supermercado, con desinfectante.

- Vuelva a lavar sus manos con agua y jabón, luego de desempacar los alimentos.

- Para que las frutas y verduras duren más tiempo, puede guardar en trozos pequeños, dentro de bolsas en el congelador.

- Prefiera alimentos no perecibles sin gluten y aquellos que de forma natural no contienen esta proteína como legumbres, arroz, quínoa, frutos secos (maní, nueces, almendras), carnes, pescados, huevos, frutas, verduras, entre otros. Los alimentos procesados libres de gluten tienen una menor calidad nutricional frente a sus similares con gluten.

- Evite la contaminación cruzada (o contacto directo) con gluten. Prepare primero sus alimentos. No comparta bombilla, vasos o cubiertos con otra persona.

- El pan sin gluten puede congelarse para que dure más tiempo. Por el contrario, puede comprar premezclas y/o harinas sin gluten para hacer su propio pan u otras preparaciones.

- Si sobra comida preparada, se recomienda congelarla identificando qué preparación es y la fecha de elaboración.

- Se recomienda la vacunación de la población de riesgo contra las infecciones habituales en invierno tales como la influenza y las bacterias productoras de neumonía, como el neumococo, ya que su aplicación reduciría la posibilidad de sufrir varias infecciones en forma simultánea. La EC por ser de carácter autoinmune se encuentra dentro del grupo de riesgo al que se recomienda la vacunación según la campaña Influenza MINSAL $2020^{25}$. $\mathrm{Su}$ aplicación para los pacientes con EC debería priorizarse especialmente en los grupos de riesgo tales como, mayores de 65 años, embarazadas, niños y niñas desde los 6 meses hasta 5to básico y personas con otras patologías crónicas. Consulte previamente con su médico tratante y/o su centro de salud habitual respecto sobre la posibilidad de su vacunación.

- Para más información y educación continua visite la página http://www.fundacionconvivir.cl/

\section{Conclusiones}

No se ha descrito hasta el momento que el padecer EC incremente el riesgo de contagio por SARSCoV-2 ni la gravedad de la enfermedad. Los adultos mayores con condiciones médicas preexistentes son más susceptibles a presentar cuadros graves. Por lo tanto, es razonable pensar que celíacos mayores de 65 años de sexo masculino con hipertensión, diabetes, obesidad, tabaquismo, enfermedades pulmonar, cardiovascular y renal, formarían parte de este grupo de riesgo, por lo cual deben seguir rigurosamente las medidas de prevención y distanciamiento social. Aunque la EC es autoinmune, no debe ser considerada como una condición de inmunodepresión, excepto casos excepcionales durante el curso de una EC grave mal controlada o refractaria. La adherencia estricta a la DLG mejora la respuesta intestinal y junto con un adecuado control médico y nutricional aseguraría a los enfermos celíacos estar mejor preparados para esta pandemia. 


\section{Artículo de Revisión}

\section{Referencias}

1.- Lebwohl B, Sanders DS, Green PHR. Coeliac disease. Lancet. 2018;391:70-81

2.- Miquel JF, Weitz JC. Enfermedad Celíaca. Capítulo 19: 178-185. Gastroenterología y Hepatología Clínica. Editorial Mediterráneo. Tercera Edición 2020.

3.- Ministerio de Salud de Chile. Resumen ejecutivo y equipo de trabajo. Encuesta Nacional de Salud 2009-2010.

4.- Jabri B, Sollid LM. Tissue-mediated control of immunopathology in coeliac disease. Nat Rev Immunol. 2009;9:85870.

5.- Wucherpfennig KW. Mechanisms for the induction of autoimmunity by infectious agents. J Clin Invest. 2001;108:1097-104.

6.- Kårhus LL, Gunnes N, Størdal K, Bakken IJ, Tapia G, Stene LC, Håberg SE, Mårild K. Influenza and risk of later celiac disease: a cohort study of 2.6 million people. Scand J Gastroenterol. 2018;53:15-23

7.- Mormile R. Severe influenza symptoms in celiac disease: implications of CD103dendritic cells? Int J Colorectal Dis. 2016;31:1551.

8.- Ungaro RC, Sullivan T, Colombel J-F, Patel G, What Should Gastroenterologists and Patients Know About COVID-19?, Clinical Gastroenterology and Hepatology 2020 https://doi. org/10.1016/j.cgh.2020.03.020 [Epub ahead of print]

9.- Lebwohl B, Green PHR, Söderling J, Roelstraete B, Ludvigsson JF. Association Between Celiac Disease and Mortality Risk in a Swedish Population. JAMA. 2020;323:1277-85.

10.- Wang D, Hu B, Hu C, Zhu F, Liu X,
Zhang J, et al. Clinical Characteristics of 138 Hospitalized Patients With 2019 Novel Coronavirus-Infected Pneumonia in Wuhan, China. Jama 2020 323:10619.

11.- Guan W-J, Ni Z-Y, Hu Y, Liang WH, Ou CQ, He JX, et al. Clinical Characteristics of Coronavirus Disease 2019 in China. N Engl J Med 2020. doi: 10.1056/ NEJMoa2002032 [Epub ahead of print].

12.- Gu J, Han B, Wang J. COVID-19: Gastrointestinal manifestations and potential fecal-oral transmission. Gastroenterology 2020. doi: 10.1053/j. gastro.2020.02.054 [Epub ahead of print].

13.- Jin X, Lian JS, Hu JH, Gao J, Zheng L, Zhang YM, et al. Epidemiological, clinical and virological characteristics of 74 cases of coronavirus-infected disease 2019 (COVID-19) with gastrointestinal symptoms. Gut 2020. doi: 10.1136/ gutjnl-2020-320926. [Epub ahead of print].

14.- Cheung KS, Hung IFN, Chan PPY, Lung KC, Tso E, Liu R, et al. Gastrointestinal Manifestations of SARS-CoV-2 Infection and Virus Load in Fecal Samples from the Hong Kong Cohort and Systematic Review and Meta-analysis. Gastroenterology 2020 doi: 10.1053/j. gastro.2020.03.065 [Epub ahead of print]

15.- Ministerio de Salud de Chile. SITUACIÓN EPIDEMIOLÓGICA COVID-19. CHILE. Consultado el 22 de abril de 2020. Disponible en: https:// www.minsal.cl/nuevo-coronavirus-2019ncov/informe-epidemiologico-covid-19/

16.- Xiao F, Tang M, Zheng X, Liu Y, Li X, Shan $H$. Evidence for Gastrointestinal Infection of SARS-CoV-2. Gastroenterology 2020 DOI: https://doi. org/10.1053/j.gastro.2020.02.055 [Epub ahead of print]
17.- Jordan RE, Adab P, Cheng KK. Covid-19: risk factors for severe disease and death. BMJ. 2020;368:m1198

18.- D'Antiga L. Coronaviruses and immunosuppressed patients. The facts during the third epidemic. Liver Transpl 2020. doi:10.1002/1t.25756.

19.- Shariati A, Aslani HR, Shayesteh MRH, Taghipour A, Nasser A, Safari H, et al. Are Viruses and Parasites Linked to Celiac Disease? A Question that Stil has no Definite Answer. Curr Pharm Biotechnol. 2019;20:1181-93.

20.- CELIAC DISEASE FOUNDATION https://celiac.org/celiac-disease-andcovid-19/\# (Consultado el 26 abril de 2020).

21.- Sociedad Española de Enfermedad Celíaca (SEEC). http://www.seec.es/ (Consultado el 26 de abril de 2020).

22.- Caio G, Volta U, Sapone A, Leffler DA, De Giorgio R, Catassi C, et al. Celiac disease: a comprehensive current review. BMC Med. 2019;17:142. Published 2019 Jul 23. doi:10.1186/s12916-0191380-z

23.- Cowling BJ, Ali ST, Ng TWY, Tsang TK, Li JCM, Fong MW, et al. Impact assessment of non-pharmaceutical interventions against coronavirus disease 2019 and influenza in Hong Kong: an observational study. Lancet Public Health. 2020 Apr 17. DOI:https://doi. org/10.1016/S2468-2667(20)30090-6 [Epub ahead of print].

24.- Desai AN, Aronoff DM. Food Safety and COVID-19. JAMA. 2020;10.1001/ jama.2020.5877. doi:10.1001/ jama.2020.5877.

25.- Campaña Influenza 2020 MINSAL https://www.minsal.cl/campanainfluenza-2020/ (Consultado el 25 de abril de 2020). 